\title{
Ambient BackCom in Beyond 5G NOMA Networks: A Multi-Cell Resource Allocation Framework
}

\author{
Wali Ullah Khan, Fida Hussain Memon, Kapal Dev, Muhammad Awais Javed, \\ Dinh-Thuan Do, and Nawab Muhammad Faseeh Qureshi
}

\begin{abstract}
The research on non-orthogonal multiple access (NOMA) is extensively used to increase the capacity of beyond fifth-generation networks. The recent merger of NOMA with ambient backscatter communication (BackCom), though opens new possibilities of massive connectivity, poses several challenges in dense wireless networks. One of such challenges is the performance degradation of ambient BackCom in multicell NOMA networks under the effect of inter-cell interference. Driven by providing an efficient solution to the issue, this article proposes a new resource allocation framework that uses the dual theory approach. Specifically, the sum-rate of the multi-cell network having backscatter tags and NOMA user equipments is maximized by formulating a joint optimization problem. To find the efficient base station transmit power and backscatter reflection coefficient in each cell, the original problem is first divided into two subproblems and then derived the closed-form solutions. A comparison with the orthogonal multiple access (OMA) ambient BackCom and pure NOMA transmission has been provided. Simulation results of proposed NOMA ambient BackCom indicate a significant improvement over the OMA ambient BackCom and pure NOMA in terms of sum-rate gains.
\end{abstract}

Index Terms-Beyond fifth-generation (5G), non-orthogonal multiple access (NOMA), ambient backscatter communication (BackCom), spectrum efficiency optimization.

\section{INTRODUCTION}

Nex generation wireless networks demand high spectral efficiency, low energy consumption, and increased connectivity [1], [2]. Non-orthogonal multiple access (NOMA) is a potential solution to meet these demands in beyond fifth-generation (5G) cellular networks [3], [4]. Its spectrum efficiency over the conventional orthogonal multiple access (OMA) solutions has made it popular among both industry and academia [5], [6]. Multiple users in a system using NOMA technique can access the same spectrum resources at one time [7]. The signals of these users can be successfully multiplexed at transmitter using superposition coding technique. The decoding process takes place at the receivers using successive interference cancellation (SIC) technique [8]. As a favorable technology to improve both the system capacity and user access, the applications

Wali Ullah Khan is with the Interdisciplinary Center for Security, Reliability and Trust (SnT), University of Luxembourg, 1855 Luxembourg City, Luxembourg (Emails: waliullah.khan@uni.lu)

Fida Hussain Memon is with the Department of Electrical Engineering Sukkur IBA University, Pakistan.

Kapal Dev is with the Department of Institute of Intelligent Systems, University of Johannesburg, South Africa.

Muhammad Awais Javed is with the Department of Electrical and Computer Engineering, COMSATS University Islamabad, 44000 Islamabad, Pakistan.

Dinh-Thuan Do is with the Department of Electrical and Computer Engineering, The University of Texas at Austin, TX 78712, USA.

Nawab Muhammad Faseeh Qureshi is with the Department of Computer Education, Sungkyunkwan University, Seoul, Korea. of NOMA have been explored for cognitive radio (CR) [9], millimeter-wave (mmWave) [10], and massive multiple-inputand-multiple-output (MIMO) heterogeneous networks [11]. Besides, some other studies indicate applications of NOMA for grant-free access [12], physical layer security [13], vehicular communication [14] and mobile-edge computing [15]. The rapid evolution of NOMA techniques has now led to the point where researchers have started exploring their applications in backscatter communication.

Lately, backscatter communication (BackCom) is emerging as a power- and spectrum-efficient technology to enable massive connectivity [16]. Generally, BackCom consists of two parts, i.e., an RF source/ reader and a backscatter tag [17]. For the case of mono-static BackCom, the RF source and reader are collocated. The carrier emitted by the RF source is reflected by the backscatter tag. The reflected signal is again received by the reader at the RF source which decodes the information. This principle of BackCom is used in the radio frequency identification (RFID) tags. Nonetheless, several other customized solutions of BackCom have been proposed as a result of the gradual evolution of this technology [18]. One of the more improved versions is bistatic BackCom, which allow efficient communication when RF source and reader are not co-located. Another improved variant is ambient BackCom, which provides a more robust spectrum utilization by removing the need for a dedicated RF source [19]. Specifically, the ambient BackCom enable the exploitation of available RF signals. By proper implementation, the ambient BackCom removes the need of a dedicated RF source [20]. However, this also brings some complexity in the hardware design of the backscatter tag and the receiver [21]. Fig. 1 shows different types of BackCom systems.

\section{A. Literature Review}

Recently, researches have been studied BackCom in both OMA and NOMA networks. For example, the authors in [22] have used an optimization technique to enhance the throughput of OMA BackCom network. Guo et al. [23] have optimized the successive decoding rate of OMA BackCom system. The work in [24] has investigated the trade-off between energy and spectral efficiency of BackCom system. Jameel et al. [25] have studied the applications of BackCom in health-care system. The authors of [26] have proposed a technique for physical layer security of cognitive BackCom system. Tao et al. [27] have proposed a new interference cancellation technique for OMA BackCom to investigate the bit error rate of the system. Reference [28] has provided an efficient resource management 


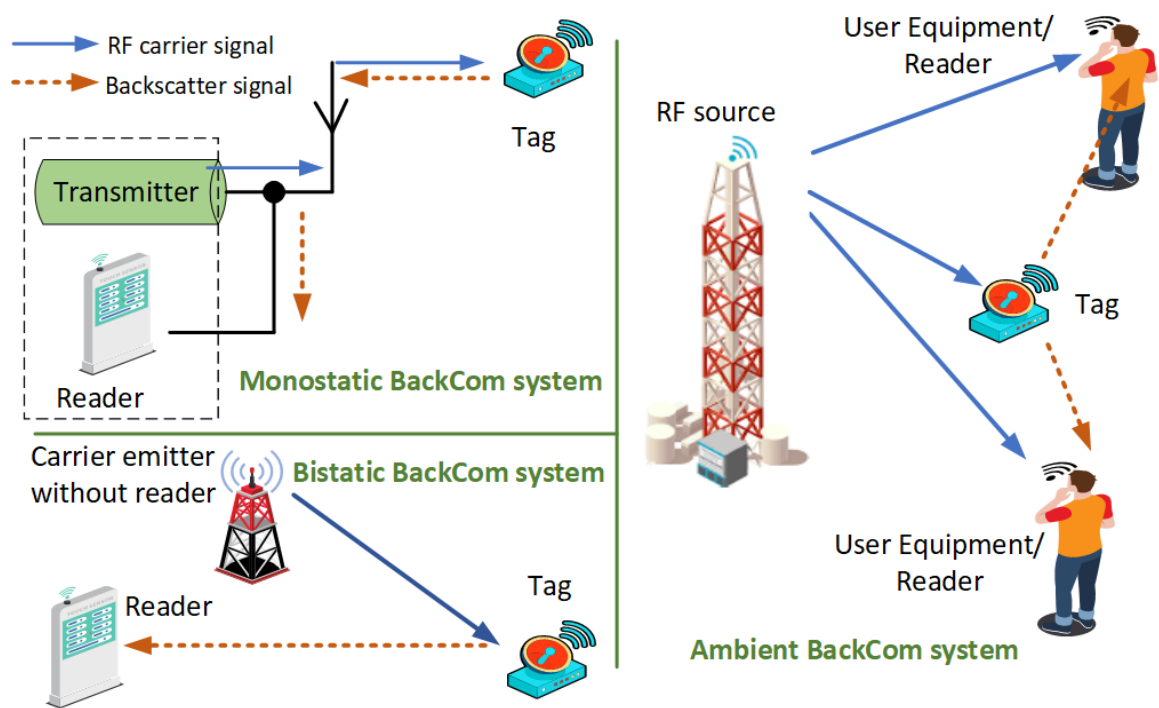

Fig. 1: Types of BackCom systems.

approach to improve the energy efficiency of mobile edge computing BackCom network. Wang et al. [29] have designed an energy efficiency BackCom system by optimizing the backscatter tag's reflection coefficient. Moreover, the work in [30] has optimized the power splitting and time switching for BackCom to investigate the rate-energy trade-off. Besides, the researches of [31]-[33] have also studied various applications of OMA BackCom in wireless networks.

Despite extensive research efforts, the studies on NOMA BackCom are still in their nascent stage. In this regard, most of the studies evaluate the performance evaluation aspect of such systems. For instance, the authors of [34] have provided the outage analysis of NOMA BackCom system. Guo et al. [35] have used NOMA for both fading-less and faded BackCom scenarios. Besides this, they also provided closed-form expressions of successfully decoded bits. In [36], a new optimization framework has proposed for unmanned aerial vehicles (UAVs) and NOMA BackCom to reduce the power consumption of UAVs while improving the number of successive decided bits. For the case of multiple-input single-input, the authors of [37] have investigated the outage analysis of NOMA BackCom system. In another study [38], Li et al. have focused on the secrecy-rate of BackCom network. To improve the sum-rate, the work in [39] has jointly optimized the reflection coefficient and time allocation of NOMA BackCom network. In a similar study [40], the authors have provided a new approach to enhance the spectral efficiency and outage probability of the NOMA BackCom system. Zhang et al. [41] have explored the outage analysis of BackCom system. Reference [42] studied a secrecy optimization problem of BackCom in multi-cell NOMA network. To enhance the physical layer security, the authors of [43] have calculated the transmit power and reflection coefficient in NOMA BackCom system. Of late, Jameel et al. [44] have proposed a reliable power allocation scheme for interference management in backscatter heterogeneous networks using reinforcement learning. Moreover, the studies of [45], [46] have optimized the spectral efficiency of NOMA
BackCom system under the perfect and imperfect channel state information (CSI) assumption. Of late, Khan et al. [47] have provided a new BackCom framework for NOMA vehicularto-everything network.

\section{B. Motivation and Contributions}

It is evident by the aforementioned discussion that NOMA BackCom is slowly maturing as a feasible technology for the massive connectivity of low-powered devices. It can also be observed that most of the researches have considered single cell scenarios. However, there is still room for improvement before the widespread adoption of BackCom. In this regard, it is critical to evaluate the performance of NOMA BackCom in the multi-cell environment. Such studies may also help in mitigating the impact of inter-cell interference on NOMA BackCom through robust optimization techniques. In this backdrop, our article provides following novel contributions:

1) A multi-cell NOMA BackCom architecture has been considered, where user equipments in each cell communicate over the same frequency band using NOMA protocol. The backscatter tag utilizes the superimposed RF signal to reflect its data to nearby NOMA users.

2) An optimization framework has been developed to maximize the sum-rate of the network. The original nonconvex optimization problem has been decoupled into two subproblems for finding the optimal base station (BS) transmit power and backscatter reflection coefficient. The subproblems are then solved using the dual decomposition approach.

3) A comprehensive comparison of the proposed NOMA BackCom framework has been provided with pure NOMA and OMA BackCom frameworks. Results show the significant improvement in the sum-rate of the system using proposed NOMA BackCom over the benchmark pure NOMA as well as conventional OMA systems. 


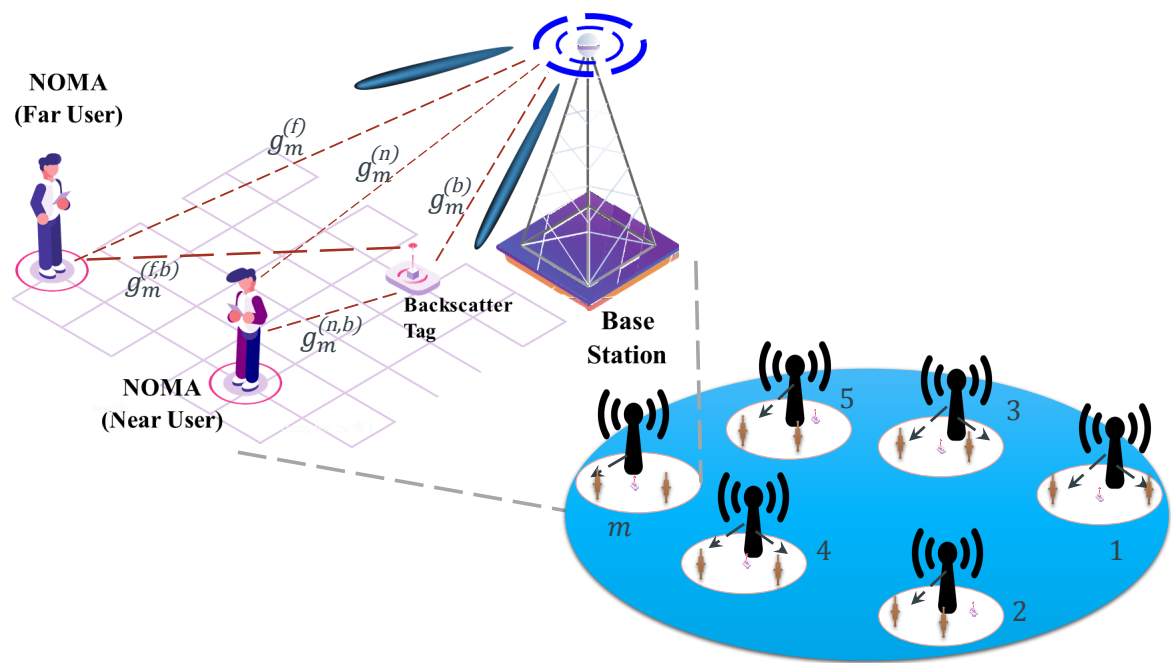

Fig. 2: System model of multi-cell NOMA Ambient BackCom network.

The remainder of the article is structured as follows. Section II discusses the system model of multi-cell network. Section III provides the problem formulation and various steps of the BS power allocation and backscatter reflection coefficient optimization techniques. In Section IV, the performance evaluation is presented along with a discussion on results. Section V highlights the conclusions of the work and possible future research directions.

\section{System And Channel Models}

A downlink multi-cell BackCom ${ }^{1}$ network is considered as shown in Fig. 2. In the considered model, the BS is assumed to be located at the center of each cell and communicates with two user equipments using NOMA protocol. Practically, user equipments in the same cell have various channel conditions, thus, we consider that the user equipment located near to the BS has stronger channel gain over the user equipment located far from the BS [48]. For better spectrum utilization, we also assume that all the BSs are sharing the same frequency [49]. The set of BSs can be denoted as $\mathcal{M}=\{m \mid 1,2,3, \ldots, M\}$, where $m$ index $m$-th BS. The proposed system also consists of $B$ uniformly distributed backscatter tags, where $b$ index $b$-th backscatter tag and $\mathcal{B}=\{b \mid 1,2,3, \ldots, B\}$ represents the set of backscatter tags, respectively. The information of user equipments in each cell is available at their serving BS. A single-input single-output case is considered, where all the devices are equipped with omni-directional antenna. A backscatter tag in each cell also simultaneously receives the RF signal from the BS. It harvests some energy from the RF signal and then reflects it towards user equipments by adding its own information. According to the downlink NOMA protocol, near user equipment can apply SIC to subtract the information of weak user equipment before decoding its own information [38], [50]. In the end, the far user equipment will not apply SIC and decode its information by treating the signal of near user equipment as noise. The superimposed signal of $m$-th BS

\footnotetext{
${ }^{1}$ The term BackCom in this paper refers to ambient BackCom; however, the full name is omitted here for the sake of simplicity.
}

for near user equipment and far user equipment is denoted as $x_{m}$ and can be given as:

$$
x_{m}=\sqrt{P_{m} \omega_{m}} z_{m}^{(n)}+\sqrt{P_{m}\left(1-\omega_{m}\right)} z_{m}^{(f)},
$$

where $z_{m}^{(n)}$ and $z_{m}^{(f)}$ are the transmitted symbols of $m$-th BS for near user equipment (denoted as $n$-th) and far user equipment (stated as $f$-th), respectively. Furthermore, $P_{m}$ is the power budget of $m$-th BS and $0<\omega_{m}<0.5$ represents the power allocation coefficient of $m$-th BS. According to powerdomain NOMA, the $m$-th BS allocates more transmit power to the $f$-th user equipment compared to the $n$-th user equipment to ensure the successful operation of SIC at receiver side [51]. As discussed earlier, the $b$-th backscatter tag modulates $s_{m}$ symbol, i.e., $\mathbb{E}\left[\left|s_{m}\right|^{2}\right]=1$, and reflect it towards user equipments. Thus, $n$-th user equipment receives the following signal:

$$
\begin{aligned}
y_{m}^{(n)} & =g_{m}^{(n)} x_{m}+\sqrt{\alpha_{m}^{(b)}} g_{m}^{(b)} x_{m} g_{m}^{(n, b)} s_{m} \\
& +\sum_{m^{\prime}=1, m^{\prime} \neq m}^{M} h_{m^{\prime}}^{(n)} \sqrt{P_{m^{\prime}}} x_{m^{\prime}}+\beta_{m}^{(n)},
\end{aligned}
$$

where $g_{m}^{(b)}$ and $g_{m}^{(n)}$ are the channel gains from the BS to near user equipment and backscatter tag while $g_{m}^{(n, b)}$ is the channel gain from the backscatter tag to near user equipment. Similarly, $h_{m^{\prime}}^{(n)}$ is the interference channel gain from the neighboring $\mathrm{BS}$ to near user equipment, $P_{m^{\prime}}$ denotes the interference power of neighboring BS, and $x_{m^{\prime}}$ represents the data symbol of neighboring BS. Moreover, the term $\alpha_{m}^{(b)}$ represnts the reflection coefficient of the backscatter tag and $\beta_{m}^{(n)}$ is the zero mean additive white Gaussian noise (AWGN) with variance $\sigma^{2}$.

Considering the effect of double fading, the signal of backscatter tag is very weaker compared to the signal of BS. Therefore, $n$-th user equipment need to apply SIC to decode and remove its own signal as well as the signal of $f$-th user equipment from $m$-th BS before decoding its desired signal from the backscatter tag. Thus, the signal to interference plus 
noise ratio (SINR) at $n$-th user equipment for decoding the signal of $f$-th user equipment can be stated as:

$$
\begin{aligned}
& \gamma_{m}^{(n, f)}= \\
& \frac{\left|g_{m}^{(n)}\right|^{2} P_{m}\left(1-\omega_{m}\right)}{P_{m} \omega_{m}\left(\left|g_{m}^{(n)}\right|^{2}+\left|g_{m}^{(n, b)}\right|^{2} \alpha_{m}^{(b)}\left|g_{m}^{(b)}\right|^{2}\right)+\ell_{m}^{(n)}+\sigma^{2}},
\end{aligned}
$$

where $\ell_{m}^{(n)}=\sum_{m^{\prime}=1, m^{\prime} \neq m}^{M} P_{m^{\prime}}\left|h_{m^{\prime}}^{(n)}\right|^{2}$ is the interference from other BSs. After removing $z_{m}^{(f)}$, the $n$-th user equipment decodes its own signal. Therefore, the SINR of $n$-th user equipment to decode its own signal can be given as:

$$
\gamma_{m}^{(n, n)}=\frac{\left|g_{m}^{(n)}\right|^{2} P_{m} \omega_{m}}{\left(\left|g_{m}^{n, b}\right|^{2} \alpha_{m}^{(b)}\left|g_{m}^{(b)}\right|^{2}\right)\left(1-\omega_{m}\right) P_{m}+\ell_{m}^{(n)}+\sigma^{2}},
$$

Finally, $n$-th user equipment decodes the signal received from the $b$-th backscatter tag. Thus, the SINR of $n$-th user equipment for decoding the signal from backscatter tag can be derived as:

$$
\gamma_{m}^{n, b}=\frac{\left|g_{m}^{(n, b)}\right|^{2} \alpha_{m}^{(b)}\left|g_{m}^{(b)}\right|^{2}\left(P_{m} \omega_{m}+P_{m}\left(1-\omega_{m}\right)\right)}{\ell_{m}^{(n)}+\sigma^{2}} .
$$

Accordingly, the received signal of $n$-th user equipment in $m$-th cell can be given as:

$$
\begin{aligned}
y_{m}^{(f)} & =g_{m}^{(f)} x_{m}+\sqrt{\alpha_{m}^{(b)}} g_{m}^{(b)} x_{m} g_{m}^{(f, b)} s_{m} \\
& +\sum_{m^{\prime}=1, m^{\prime} \neq m}^{M} h_{m^{\prime}}^{(f)} \sqrt{P_{m^{\prime}}} x_{m^{\prime}}+\beta_{m}^{(f)},
\end{aligned}
$$

where $g_{m}^{(f)}$ and $g_{m}^{(f, b)}$ show the channel gains from $m$-th BS to $f$-th user equipment and from $b$-th backscatter tag to $f$ th user equipment associated with $m$-th BS, respectively. As such, $h_{m^{\prime}}^{(f)}$ is the interference channel gain from $m^{\prime}$-th BS to $f$-th user equipment in $m$-th cell. Finally, the term $\beta_{m}^{(f)}$ is the zero mean AWGN with variance $\sigma^{2}$. It is important to note that $f$-th user equipment in $m$-th cell decodes its own signal with the interference of $n$-th user equipment and $b$-th backscatter tag. Thus, the SINR of $f$-th user equipment to decode its desired signal can be stated as:

$$
\begin{aligned}
& \gamma_{m}^{(f, f)}= \\
& \frac{\left|g_{m}^{(f)}\right|^{2} P_{m}\left(1-\omega_{m}\right)}{P_{m} \omega_{m}\left(\left|g_{m}^{(f)}\right|^{2}+\left|g_{m}^{(f, b)}\right|^{2} \alpha_{m}^{(b)}\left|g_{m}^{(b)}\right|^{2}\right)+\ell_{m}^{(f)}+\sigma^{2}},
\end{aligned}
$$

where $\ell_{m}^{(f)}=\sum_{m^{\prime}=1, m^{\prime} \neq m}^{M} P_{m^{\prime}}\left|h_{m^{\prime}}^{(f)}\right|^{2}$ denotes the interference from all other BSs.

\section{Problem Formulation and Proposed Solutions}

We discuss different formulation steps of sum-rate maximization problem followed by the closed-form solutions of BS transmit power and backscatter tag reflection coefficient in this section. Then, we also present the proposed algorithm and complexity analysis.

\section{A. Problem Formulation}

In this work, we evaluate the system performance by maximizing the sum-rate of multi-cell BackCom network. In particular, the rate of user equipments from BSs and from the backscatter tags to user equipments. The total rate of backscatter multi-cell network can be then formulated as:

$$
R_{t o t}=\sum_{m=1}^{M}\left(R_{m}^{(n, n)}+R_{m}^{(f, f)}+R_{m}^{(n, b)}\right), \forall n, \forall f, \forall b,
$$

where $R_{m}^{(n, n)}, R_{m}^{(f, f)}$, and $R_{m}^{(n, b)}$ are the rates of $n$-th user equipment from $m$-th $\mathrm{BS}$, the rate of $f$-th user equipment from the $m$-th BS, and the rate of $n$-th user equipment from $b$-th backscatter tag in $m$-th cell ${ }^{2}$, respectively. Moreover, these rates can be given as follow:

$$
\begin{aligned}
& R_{m}^{(n, n)}=\log _{2}\left(1+\gamma_{m}^{(n, n)}\right), \\
& R_{m}^{(f, f)}=\log _{2}\left(1+\gamma_{m}^{(f, f)}\right), \\
& R_{m}^{(n, b)} \approx \log _{2}\left(1+\gamma_{m}^{(n, b)}\right) .
\end{aligned}
$$

The target of total rate maximization can be attained by user equipment's transmit power optimization as well as the optimization of reflection coefficient of backscatter tags. This optimization framework is subject to the reflection coefficients of backscatter tags and the transmit power of BSs according to the NOMA protocol ${ }^{3}$. Mathematically, an optimization problem can be formulated to maximize the sum-rate of multicell BackCom network as:

$$
\begin{array}{ll} 
& \text { OP } \quad \underset{\omega_{m}, \alpha_{m}^{(b)}}{\operatorname{Maximize}} R_{\text {tot }} \\
\text { s.t. } \quad & C 1: 0 \leq \alpha_{m}^{(b)} \leq \alpha_{\max }, \forall m, \forall b, \\
& C 2: P_{m} \omega_{m} \leq P_{m}\left(1-\omega_{m}\right), \forall m, \\
& C 3: 0 \leq P_{m} \leq P_{\max }, \forall m, \\
& C 4: 0 \leq \omega_{m} \leq 0.5, \forall m,
\end{array}
$$

where constraint $C 1$ limits the reflection coefficient between 0 and $\alpha_{\max }$, where $\alpha_{\max }$ is the maximum coefficient power the $b$-th backscatter tag can reflect. Constraints $C 2$ and $C 4$ bound BSs to transmit power according NOMA principle. In addition, $C 3$ limits each BS transmit power, where $P_{\max }$ shows the BS's maximum transmission power threshold.

The above formulated optimization problem of sum-rate is non-convex [54]. Moreover, it also coupled on BS power allocation and backscatter tag reflection coefficient selection, which is very complex and difficult to solve. The complexity of the original problem is reduced by first decoupling it into two subproblems for allocating optimal power at BSs and finding optimal reflection coefficient at backscatter tags. And then provide suboptimal techniques to get the efficient solutions.

${ }^{2}$ Calculting the exact capacity of BackCom is difficult in practice [52]. Thus, a common approach is to approximate the maximum achievable capacity [22], [53].

${ }^{3}$ Due to the weak signal of BackCom [34], the interference from backscatter tag to far user equipment is almost non-existent and brings negligible change in its performance. 


$$
\begin{aligned}
& \frac{\partial \mathcal{L}(.)}{\partial \omega_{m}}=\frac{\partial}{\partial \alpha}\left[\log _{2}\left(1+\frac{\left|g_{m}^{(n)}\right|^{2} P_{m}\left(1-\omega_{m}\right)}{\left|g_{m}^{(n)}\right|^{2} P_{m} \omega_{m}+\left|g_{m}^{(n, b)}\right|{ }^{2} \alpha_{m}^{(b)}\left|g_{m}^{(b)}\right|^{2} P_{m}+\ell_{m}^{(n)}+\sigma^{2}}\right)\right. \\
&+\log _{2}\left(1+\frac{\left|g_{m}^{(f)}\right|^{2} P_{m}\left(1-\omega_{m}\right)}{\left|g_{m}^{(f)}\right|^{2} P_{m} \omega_{m}+\left|g_{m}^{(f, b)}\right|^{2} \alpha_{m}^{(b)}\left|g_{m}^{(b)}\right|^{2} P_{m}+\ell_{m}^{(f)}+\sigma^{2}}\right) \\
&\left.+\log _{2}\left(1+\frac{\left|g_{m}^{(n, b)}\right|^{2} \alpha_{m}^{(b)}\left|g_{m}^{(b)}\right|^{2} P_{m}}{\ell_{m}^{(n)}+\sigma^{2}}\right)-\mu_{m}\left(P_{m} \omega_{m}-P_{m}\left(1-\omega_{m}\right)\right)-\eta_{m}\left(\omega_{m}-0.5\right)\right]=0 . \\
& \frac{\partial \mathcal{L}(.)}{\partial \omega_{m}}= \frac{\partial}{\partial \omega_{m}}\left[\log _{2}\left(\left|g_{m}^{(n)}\right|^{2} P_{m}\left(1-\omega_{m}\right)\right)-\log _{2}\left(\left|g_{m}^{(n)}\right|^{2} P_{m} \omega_{m}+\left|g_{m}^{(n, b)}\right|^{2} \alpha_{m}^{(b)}\left|g_{m}^{(b)}\right|^{2} P_{m}+\ell_{m}^{(n)}+\sigma^{2}\right)\right. \\
&+ \log _{2}\left(\left|g_{m}^{(f)}\right|^{2} P_{m}\left(1-\omega_{m}\right)\right)-\log _{2}\left(\left|g_{m}^{(f)}\right|^{2} P_{m} \omega_{m}+\left|g_{m}^{(f, b)}\right|^{2} \alpha_{m}^{(b)}\left|g_{m}^{(b)}\right|^{2} P_{m}+\ell_{m}^{(f)}+\sigma^{2}\right) \\
&+\left.\log _{2}\left(\left|g_{m}^{(n, b)}\right|^{2} \alpha_{m}^{(b)}\left|g_{m}^{(b)}\right|^{2} P_{m}\right)-\log _{2}\left(\ell_{m}^{(n)}+\sigma^{2}\right)-\mu_{m}\left(P_{m} \omega_{m}-P_{m}\left(1-\omega_{m}\right)\right)-\eta_{m}\left(\omega_{m} P_{m}-0.5\right)\right]=0 . \\
& \frac{\partial \mathcal{L}(.)}{\partial \omega_{m}}=\frac{\left|g_{m}^{(f)}\right|^{2} P_{m}}{\left|g_{m}^{(n)}\right|^{2} P_{m} \omega_{m}}-\frac{\left|g_{m}^{(f)}\right|^{2} P_{m} \omega_{m}+\left|g_{m}^{(f, b)}\right|^{2} \alpha_{m}^{(b)}\left|g_{m}^{(b)}\right|^{2} P_{m}+\ell_{m}^{(f)}+\sigma^{2}}{}-\mu_{m} P_{m}-\eta_{m}=0 . \\
& \frac{\left(\left|g_{m}^{(n)}\right|^{2} P_{m}\right)\left(\left|g_{m}^{(f)}\right|^{2} P_{m} \omega_{m}+\left|g_{m}^{(f, b)}\right|^{2} \alpha_{m}^{(b)}\left|g_{m}^{(b)}\right|^{2} P_{m}+\ell_{m}^{(f)}+\sigma^{2}\right)-\left(\left|g_{m}^{(f)}\right|^{2} P_{m}\right)\left(\left|g_{m}^{(f)}\right|^{2} P_{m} \omega_{m}\right)}{\left(\left|g_{m}^{(n)}\right|{ }^{2} P_{m} \omega_{m}\right)\left(\left|g_{m}^{(f)}\right|^{2} P_{m} \omega_{m}+\left|g_{m}^{(f, b)}\right|^{2} \alpha_{m}^{(b)}\left|g_{m}^{(b)}\right|^{2} P_{m}+\ell_{m}^{(f)}+\sigma^{2}\right)}=\eta_{m} P_{m} .
\end{aligned}
$$

$$
C_{m}=-\frac{\left|g_{m}^{(n)}\right|{ }^{2}\left|g_{m}^{(f, b)}\right|^{2}\left|g_{m}^{(b)}\right|^{2} P_{m}^{2} \alpha_{m}^{(b)}+\left|g_{m}^{(n)}\right|^{2} P_{m} \ell_{m}^{(f)}+\left|g_{m}^{(n)}\right|^{2} P_{m} \sigma^{2}}{\eta_{m}} .
$$

\section{B. Power Allocation at BSs for the Given Reflection Coeffi-} cient of Backscatter Tags

Here we assume that the reflection coefficients of backscatter tags are fixed, and accordingly the subproblem (OP1) can be formulated as:

$$
\begin{aligned}
\text { OP1 } & \underset{\omega_{m}}{\operatorname{Maximize} R_{t o t}} \\
\text { s.t. } & (C 2)-(C 4),
\end{aligned}
$$

To solve the optimization problem (OP1) efficiently, we adopt dual theory [55], where the dual-problem of (OP1) can be derived as:

$$
\underset{\mu_{m}, \tau_{m}, \eta_{m}}{\operatorname{Minimize}} \Delta\left(\mu_{m}, \tau_{m}, \eta_{m}\right)
$$

where $\Delta($.$) is the dual-function while the dual variables$ include $\mu_{m}, \tau_{m}$, and $\eta_{m} . \Delta($.$) can be written as:$

$$
\Delta\left(\mu_{m}, \tau_{m}, \eta_{m}\right)=\underset{\omega_{m}}{\operatorname{Maximize}} \mathcal{L}\left(\omega_{m}, \mu_{m}, \tau_{m}, \eta_{m}\right),
$$

where $\mathcal{L}\left(\omega_{m}, \mu_{m}, \tau_{m}, \eta_{m}\right)$ represents Lagrangian function of $\mathrm{OP} 1$, and can be defined as:

$$
\begin{aligned}
\mathcal{L}(.) & =R_{\text {tot }}-\sum_{m=1}^{M} \mu_{m}\left(P_{m} \omega_{m}-P_{m}\left(1-\omega_{m}\right)\right) \\
& -\tau_{m}\left(P_{m}-P_{\max }\right)-\eta_{m}\left(\omega_{m}-0.5\right) .
\end{aligned}
$$

By expanding $R_{t o t}$, it can be written as:

$$
\begin{aligned}
\mathcal{L}(.) & =\sum_{m=1}^{M}\left(R_{m}^{(n, n)}+R_{m}^{(f, f)}+R_{m}^{(n, b)}\right) \\
& -\mu_{m}\left(P_{m} \omega_{m}-P_{m}\left(1-\omega_{m}\right)\right) \\
& -\tau_{m}\left(P_{m}-P_{\max }\right)-\eta_{m}\left(\omega_{m}-0.5\right),
\end{aligned}
$$

Now, we adopt the KKT conditions to calculate a closedform solution for $\omega_{m}$ as:

$$
\begin{aligned}
& \frac{\partial \mathcal{L}(.)}{\partial \omega_{m}}=\frac{\partial}{\partial \omega_{m}}\left[\log _{2}\left(1+\gamma_{m}^{(n, n)}\right)+\log _{2}\left(1+\gamma_{m}^{(f, f)}\right)\right. \\
& +\log _{2}\left(1+\gamma_{m}^{(n, b)}\right)-\mu_{m}\left(P_{m} \omega_{m}-P_{m}\left(1-\omega_{m}\right)\right) \\
& \left.-\tau_{m}\left(P_{m}-P_{\text {max }}\right)-\eta_{m}\left(\omega_{m}-0.5\right)\right]=0,
\end{aligned}
$$

Substituting the values of $\gamma_{m}^{(n, n)}, \gamma_{m}^{(f, f)}$, and $\gamma_{m}^{(n, b)}$ in (18), we can obtain as (19) on the top of next page. Considering the approximation of high signal to noise ratio [56] and using the log properties, (19) can be rewritten as (20) mentioned at the top of the next page.

After calculating partial derivatives of (20), we obtain (21) at the top of the next page. Now we calculate the least common multiple (LCM) of (21), and arrive at (22), written at the top of the next page. By setting $\mu_{m} P_{m}=0$, and then solving for $\omega_{m}$, it results in $\omega_{m}^{*}$ as:

$$
\omega_{m}^{*}=\left[\frac{-B_{m} \pm \sqrt{B_{m}^{2}-4 A_{m} C_{m}}}{2 A_{m}}\right]^{+},
$$




$$
\begin{aligned}
& \frac{\partial \mathcal{L}(.)}{\partial \omega_{m}}=\frac{\partial}{\partial \alpha_{m}^{(b)}}\left[\log _{2}\left(1+\frac{\left|g_{m}^{(n)}\right|^{2} P_{m}\left(1-\omega_{m}\right)}{\left|g_{m}^{(n)}\right|^{2} P_{m} \omega_{m}+\left|g_{m}^{(n, b)}\right|^{2} \alpha_{m}^{(b)}\left|g_{m}^{(b)}\right|^{2} P_{m}+\ell_{m}^{(n)}+\sigma^{2}}\right)\right. \\
& +\log _{2}\left(1+\frac{\left|g_{m}^{(f)}\right|^{2} P_{m}\left(1-\omega_{m}\right)}{\left|g_{m}^{(f)}\right|^{2} P_{m} \omega_{m}+\left|g_{m}^{(f, b)}\right|^{2} \alpha_{m}^{(b)}\left|g_{m}^{(g)}\right|^{2} P_{m}+\ell_{m}^{(f)}+\sigma^{2}}\right) \\
& \left.\left.+\log _{2}\left(1+\frac{\left|g_{m}^{(n, b)}\right|^{2} \alpha_{m}^{(b)}\left|g_{m}^{(b)}\right|^{2} P_{m}}{\ell_{m}^{(n)}+\sigma^{2}}\right)\right)-\lambda_{m}\left(\alpha_{m}^{(b)}-\alpha_{\max }\right)\right]=0 . \\
& \frac{\partial \mathcal{L}(.)}{\partial \alpha_{m}^{(b)}}=\frac{\partial}{\partial \alpha_{m}^{(b)}}\left[\log _{2}\left(\left|g_{m}^{(n)}\right|^{2} P_{m}\left(1-\omega_{m}\right)\right)-\log _{2}\left(\left|g_{m}^{(n)}\right|^{2} P_{m} \omega_{m}+\left|g_{m}^{(n, b)}\right|^{2} \alpha_{m}^{(b)}\left|g_{m}^{(b)}\right|^{2} P_{m}+\ell_{m}^{(n)}+\sigma^{2}\right)\right. \\
& +\log _{2}\left(\left|g_{m}^{(f)}\right|^{2} P_{m}\left(1-\omega_{m}\right)\right)-\log _{2}\left(\left|g_{m}^{(f)}\right|^{2} P_{m} \omega_{m}+\left|g_{m}^{(f, b)}\right|^{2} \alpha_{m}^{(b)}\left|g_{m}^{(b)}\right|^{2} P_{m}+\ell_{m}^{(f)}+\sigma^{2}\right) \\
& \left.+\log _{2}\left(\left|g_{m}^{(n, b)}\right|^{2} \alpha_{m}^{(b)}\left|g_{m}^{(b)}\right|^{2} P_{m}\right)-\log _{2}\left(\ell_{m}^{(n)}+\sigma^{2}\right)-\lambda_{m}\left(\alpha_{m}^{(b)}-\alpha_{\max }\right)\right]=0 . \\
& \frac{\partial \mathcal{L}(.)}{\partial \alpha_{m}^{(b)}}=\frac{\left|g_{m}^{(n, b)}\right|^{2}\left|g_{m}^{(b)}\right|^{2} P_{m}}{\left|g_{m}^{(n, b)}\right|^{2} \alpha_{m}^{(b)}\left|g_{m}^{(b)}\right|^{2} P_{m}}-\frac{\left|g_{m}^{(n, b)}\right|^{2}\left|g_{m}^{(b)}\right|^{2} P_{m}}{\left|g_{m}^{(n)}\right|^{2} P_{m} \omega_{m}+\left|g_{m}^{(n, b)}\right|^{2} \alpha_{m}^{(b)}\left|g_{m}^{(b)}\right|^{2} P_{m}+\ell_{m}^{n}+\sigma^{2}} \\
& -\frac{\left|g_{m}^{(f)}\right|^{2} P_{m}}{\left|g_{m}^{(f)}\right|^{2} P_{m} \omega_{m}+\left|g_{m}^{(f, b)}\right|^{2} \alpha_{m}^{(b)}\left|g_{m}^{(b)}\right|^{2} P_{m}+\ell_{m}^{(f)}+\sigma^{2}}-\lambda_{m}=0 . \\
& \frac{\left(\left|g_{m}^{(n, b)}\right|^{2}\left|g_{m}^{(b)}\right|^{2} P_{m}\right)\left(\kappa_{m}^{(n)}\right)\left(\iota_{m}^{(f)}\right)-\left(\left|g_{m}^{(n, b)}\right|^{2}\left|g_{m}^{(b)}\right|^{2} P_{m}\right)\left(\pi_{m}^{(b)}\right)\left(\iota_{m}^{(f)}\right)-\left(\left|g_{m}^{(f)}\right|^{2} P_{m}\right)\left(\pi_{m}^{(b)}\right)\left(\kappa_{m}^{(n)}\right)}{\left(\pi_{m}^{(b)}\right)\left(\kappa_{m}^{(n)}\right)\left(\iota_{m}^{(f)}\right)}=\lambda_{m}
\end{aligned}
$$

where $(\chi)^{+}=\max (0, \chi)$, and the values of $A_{m}$ and $B_{m}$ are given by

$$
\begin{gathered}
A_{m}=\left|g_{m}^{(n)}\right|^{2}\left|g_{m}^{(f)}\right|^{2} P_{m}^{2} \\
B_{m}=\left|g_{m}^{(n)}\right|^{2}\left|g_{m}^{(f, b)}\right|^{2} \alpha_{m}^{(b)}\left|g_{m}^{(b)}\right|^{2} P_{m}^{2}+\left|g_{m}^{(n)}\right|^{2} P_{m} \ell_{m}^{(f)} \\
+\left|g_{m}^{(f)}\right|^{2} P_{m} \sigma^{2}+\frac{\left|g_{m}^{(f)}\right|^{4} P_{m}^{2} \sigma^{2}-\left|g_{m}^{(n)}\right|^{2}\left|g_{m}^{(f)}\right|^{2} P_{m}^{2}}{\mu_{m} P_{m}+\eta_{m}}
\end{gathered}
$$

while the value of $C_{m}$ is provided in (26) on the next page. At the end, $\eta_{m}$ and $\mu_{m}$ are iteratively calculated as [57]:

$$
\begin{aligned}
& \eta_{m}(1+t)= \\
& \eta_{m}(t)+\delta(t)\left(P_{m} \omega_{m}-P_{m}\left(1-\omega_{m}\right)\right), \forall i, j, m, \\
& \mu_{m}(1+t)=\mu_{m}(t)+\delta(t)\left(\omega_{m}-0.5\right), \forall i, j, m,
\end{aligned}
$$

where $t$ represents the iterations and $\delta$ indicates the step size. In each index of $t$, we find optimal values of $\mu_{m}^{*}$ and $\eta_{m}^{*}$ using the computed value of $\omega_{m}$. Then, in further iterations, the values of $\mu_{m}^{*}$ as well as $\eta_{m}^{*}$ is utilized to obtain optimal $\omega_{m}^{*}$. This process is stopped on convergence.

\section{Calculating Reflection Coefficient at Backscatter Tags for the Available Transmit Power of BSs}

Now we find the reflection coefficient of backscatter tag for a given power allocation at BS. To do so, the original problem (OP) should be simplified as:

$$
\text { OP2 } \underset{\alpha_{m}^{(b)}}{\operatorname{Maximize}} R_{t o t}
$$$$
\text { s.t. }(C 1)
$$

This optimization problem $\mathrm{P} 2$ is convex with respect to $\alpha_{m}^{(b)}$, thus, similar to the solution of $\mathrm{P} 1$, we exploit dual theory. The corresponding dual problem can be formulated as:

$$
\underset{\lambda_{m}}{\operatorname{Minimize}} \Delta\left(\lambda_{m}\right),
$$

where $\Delta($.$) in (30) represents the dual-function and \lambda_{m}$ is a dual-variable. The dual-function can be written as:

$$
\Delta\left(\lambda_{m}\right)=\underset{\lambda_{m}}{\operatorname{Minimize}} \mathcal{L}\left(\alpha_{m}^{(b)}, \lambda_{m}\right),
$$

where $\mathcal{L}\left(\alpha_{m}^{(b)}, \lambda_{m}\right)$ represents the Lagrangian-function shown in (OP2), which can be derived as:

$$
\mathcal{L}(.)=R_{\text {tot }}-\lambda_{m}\left(\alpha_{m}^{(b)}-\alpha_{\max }\right),
$$

By expanding $R_{\text {tot }}$, it can be written as:

$$
\begin{aligned}
\mathcal{L}(.) & =\sum_{m=1}^{M}\left(R_{m}^{(n, n)}+R_{m}^{(f, f)}+R_{m}^{(n, b)}\right) \\
& -\lambda_{m}\left(\alpha_{m}^{(b)}-\alpha_{\max }\right) .
\end{aligned}
$$

To obtain closed-form expression for $\alpha_{m}^{(b)}$, KKT conditions can be used, thus resulting in following equations:

$$
\begin{aligned}
\frac{\partial \mathcal{L}(.)}{\partial \alpha_{m}^{(b)}} & =\frac{\partial}{\partial \alpha_{m}^{(b)}}\left[\log _{2}\left(1+\gamma_{m}^{(n, n)}\right)\right. \\
& +\log _{2}\left(1+\gamma_{m}^{(f, f)}\right)+\log _{2}\left(1+\gamma_{m}^{(n, b)}\right) \\
& \left.-\lambda_{m}\left(\alpha_{m}^{(b)}-\alpha_{\max }\right)\right]=0
\end{aligned}
$$

By substituting the values of $\gamma_{m}^{(n, n)}, \gamma_{m}^{(f, f)}$, and $\gamma_{m}^{(n, b)}$ in (34), we arrive at (35) written on the top of this page. Now considering the approximation for high signal to noise ratio 


$$
\begin{gathered}
D_{m}=\left|g_{m}^{(n, b)}\right|^{4}\left|g_{m}^{(f, b)}\right|^{2}\left|g_{m}^{(b)}\right|^{4} P_{m}^{3}+\frac{\left|g_{m}^{(n, b)}\right|{ }^{4}\left|g_{m}^{(b)}\right|^{4}\left|g_{m}^{(f)}\right|^{2} P_{m}^{3}}{\lambda_{m}} \\
E_{m}=\left|g_{m}^{(n, b)}\right|{ }^{4}\left|g_{m}^{(b)}\right|^{2} P_{m}^{2} \ell_{m}^{(f)}+\left|g_{m}^{(n, b)}\right|{ }^{4}\left|g_{m}^{(b)}\right|^{4} P_{m}^{2} \sigma^{2}+\left|g_{m}^{(n, b)}\right|^{2}\left|g_{m}^{(b)}\right|^{4}\left|g_{m}^{(f, b)}\right|^{2} P_{m}^{2} \sigma^{2} \\
+\frac{\left|g_{m}^{(n, b)}\right|^{2}\left|g_{m}^{(b)}\right|^{2}\left|g_{m}^{(f)}\right|^{2} P_{m}^{2}}{\lambda_{m}}+\frac{\left|g_{m}^{(n, b)}\right|^{2}\left|g_{m}^{(b)}\right|^{2}\left|g_{m}^{(f)}\right|^{2} P_{m}^{2} \ell_{m}^{(n)}}{\lambda_{m}}+\frac{\left|g_{m}^{(n, b)}\right|^{2}\left|g_{m}^{(b)}\right|^{2}\left|g_{m}^{(n)}\right|^{2} P_{m}^{2}\left|g_{m}^{(f)}\right|^{2} \omega_{m}}{\lambda_{m}} \\
+\frac{\left|g_{m}^{(n, b)}\right|{ }^{4}\left|g_{m}^{(b)}\right|^{4}\left|g_{m}^{(f)}\right|^{2} P_{m}^{2} \omega_{m}}{\lambda_{m}}-\frac{\left|g_{m}^{(n, b)}\right|^{2}\left|g_{m}^{(b)}\right|^{2}\left|g_{m}^{(f, b)}\right|^{2} P_{m}^{2} \sigma^{2}}{\lambda_{m}} \\
F_{m}=\left|g_{m}^{(n, b)}\right|^{2}\left|g_{m}^{(b)}\right|^{2}\left|g_{m}^{(f)}\right|^{2} P_{m}^{3} \omega_{m}+\left|g_{m}^{(n, b)}\right|^{2}\left|g_{m}^{(n)}\right|^{2}\left|g_{m}^{(b)}\right|^{2} P_{m}^{2} \omega_{m} \ell_{m}^{(f)} \\
-\frac{\left|g_{m}^{(n, b)}\right|^{2}\left|g_{m}^{(b)}\right|^{2}\left|g_{m}^{(f)}\right|^{2}\left|g_{m}^{(n)}\right|^{2} P_{m}^{3} \omega_{m}}{\lambda_{m}}-\frac{\left|g_{m}^{(n, b)}\right|^{2}\left|g_{m}^{(b)}\right|^{2} P_{m}^{2} \omega_{m}}{\lambda_{m}},
\end{gathered}
$$

and using the property of the log, the (35) can be formulated as (36).

We compute the partial derivatives of (36) which lead us to (37) on the top of this page. By computing the LCM of (37), we obtain (38), given at the top of this page. Here the values of $\pi_{m}^{(b)}, \kappa_{m}^{(n)}$, and $\iota_{m}^{(f)}$ in (38) can be written as:

$$
\begin{gathered}
\pi_{m}^{(b)}=\left|g_{m}^{(n, b)}\right|^{2} \alpha_{m}^{(b)}\left|g_{m}^{(b)}\right|^{2} P_{m}, \\
\kappa_{m}^{(n)}=\left|g_{m}^{(n)}\right|^{2} P_{m} \omega_{m}+\left|g_{m}^{(n, b)}\right|^{2} \alpha_{m}^{(b)}\left|g_{m}^{(b)}\right|^{2} P_{m}+\ell_{m}^{(n)}+\sigma^{2}, \\
\iota_{m}^{(f)}=\left|g_{m}^{(f)}\right|^{2} P_{m} \omega_{m}+\left|g_{m}^{(f, b)}\right|^{2} \alpha_{m}^{(b)}\left|g_{m}^{(b)}\right|^{2} P_{m}+\ell_{m}^{(f)}+\sigma^{2} .
\end{gathered}
$$

Now calculating for $\alpha_{m}^{(b)}$, it results in $\alpha_{m}^{(b) *}$ as:

$$
\alpha_{m}^{(b) *}=\left[\frac{-E_{m} \pm \sqrt{E_{m}^{2}-4 D_{m} F_{m}}}{2 D_{m}}\right]^{+},
$$

where $(v)^{+}=\max (0, v)$, and the values of $D_{m}, E_{m}$, and $F_{m}$ are respectively presented in (40), (41) and (42) on the top of the next page.

The Lagrangian multiplier $\lambda_{m}$ is iteratively updated as:

$$
\lambda_{m}(1+t)=\lambda_{m}(t)+\delta(t)\left(\alpha_{m}^{(b)}-\alpha_{\max }\right), \forall m \forall b
$$

where for each index of $t$ in (43), we find optimal $\lambda_{m}^{*}$ using the computed value of $\alpha_{m}^{(b)}$. Then, using this value of $\lambda_{m}^{*}$, we find the optimal $\alpha_{m}^{(b) *}$. This process will be terminated after convergence.

\section{Resource Allocation Algorithm and Complexity}

In this subsection, we design and discuss an iterative algorithm for the proposed solution of Section III-A and B. As depicted in Algorithm 1, all the system variables and parameters are defined first. Then, for any given value of backscatter tag reflection coefficient $\alpha_{m}^{(b)}$, we iteratively calculate the optimal value of BS power allocation coefficient $\omega_{m}^{*}$ and Lagrangian variables $\eta_{m}$ and $\mu_{m}$. Next we substitute the value of $\omega_{m}^{*}$ in subproblem (29) and calculate the efficient reflection coefficient $\alpha_{m}^{(b) *}$ of backscatter tag and $\lambda_{m}$. The above iterative process will continue until convergence criterion satisfied.

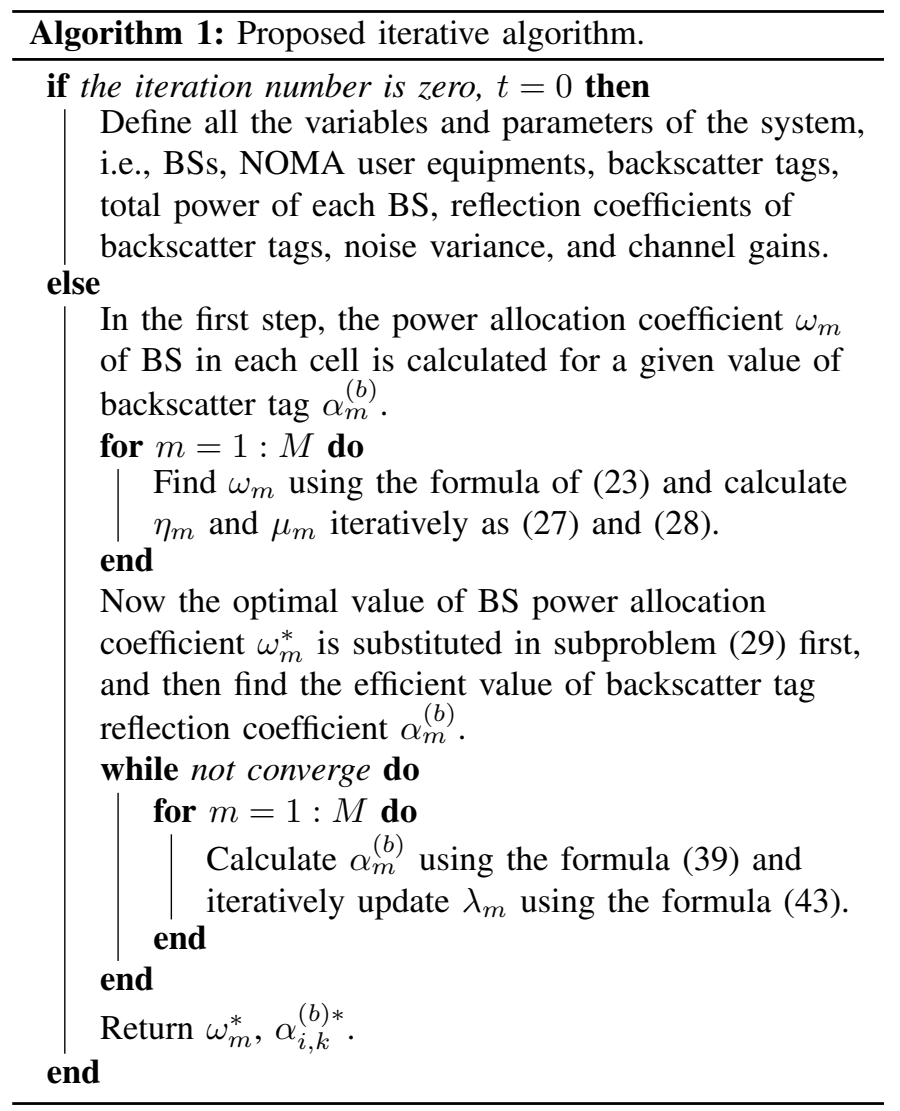

For the successful deployment of the considered BackCom system in the future, the complexity of the proposed algorithm is an important aspect.It should be noted that the computational complexity of the proposed algorithm is computed in the form of number of iterations required for the convergence. It should also be noted that the number of BSs, backscatter tags, and their associated user equipments are the factors that dictate the complexity of the proposed technique. The computational complexity of the proposed BackCom algorithm in any given iteration is calculated as $\mathcal{O}[(n+f) B M]$, where $n$ and $f$ denote the near user equipment and far user equipment. Since we have assumed that a BS communicates with two user equipments 


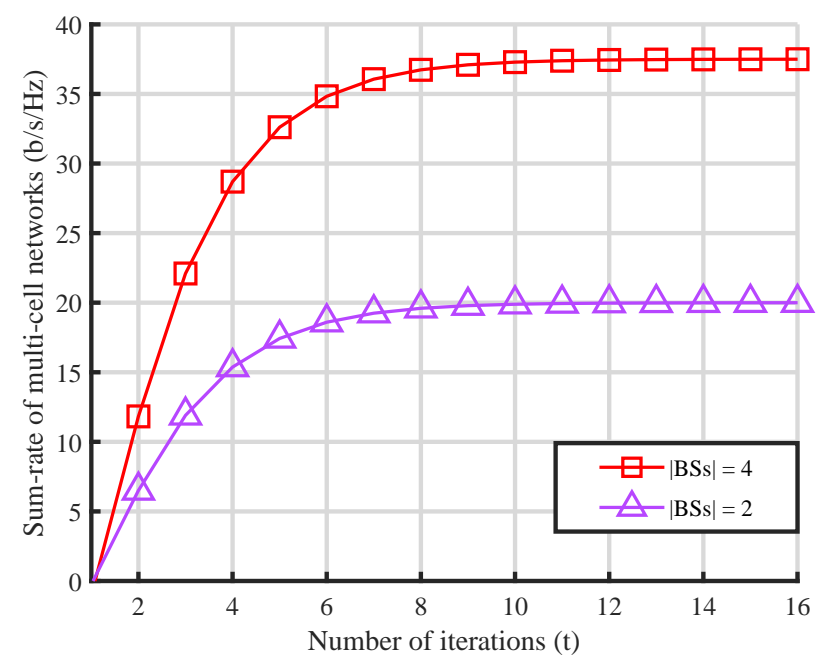

Fig. 3: Sum-rate of the proposed multi-cell NOMA BackCom versus number of iterations.

at one time, the computational complexity can also be written as $\mathcal{O}[(2) B M]$. The total number of iterations control the computational complexity of the proposed technique. If the total number iterations needed for the convergence of our algorithm is $T$. Then the total computational complexity can be expressed as $\mathcal{O}[(2) T B M]$.

\section{NumericAl Results}

In this section, we provide results for the proposed BackCom technique in multi-cell NOMA network. We perform link-level simulations in MATLAB and use Monte Carlo methods to obtain the average channel gains $\left(10^{4}\right.$ realizations). Specifically, we evaluate the system performance by sum-rate and compare three schemes, i.e., NOMA BackCom scheme, pure NOMA technique and conventional OMA BackCom technique, respectively. Here NOMA refer to the scheme without BackCom while OMA is the time division multiple access (TDMA) scheme. According to TDMA, each BS communicates to only one user equipment in a given time. Accordingly, two users can receive their signals in different time slots. The parameters used in the simulations, unless mentioned otherwise, are as follows: Rayleigh fading is used to model the channels among various devices in the system, such that $g_{m}^{(n)} \sim \mathcal{C N}\left(0, \phi_{m}^{(n)}\right), g_{m}^{(f)} \sim \mathcal{C N}\left(0, \phi_{m}^{(f)}\right), g_{m}^{(b)} \sim$ $\mathcal{C N}\left(0, \phi_{m}^{(b)}\right), g_{m}^{(n, b)} \sim \mathcal{C N}\left(0, \phi_{m}^{(n, b)}\right), g_{m}^{(f, b)} \sim \mathcal{C N}\left(0, \phi_{m}^{(f, b)}\right)$, $h_{m^{\prime}}^{(n)} \sim \mathcal{C N}\left(0, \phi_{m^{\prime}}^{(n)}\right)$, and $h_{m^{\prime}}^{(f)} \sim \mathcal{C N}\left(0, \phi_{m^{\prime}}^{(f)}\right)$, where $\phi=0.1$. Moreover, all the backscatter tags and user equipments are uniformly distributed among all cells, The number of cells is set as $M=4$, the maximum power of each $\mathrm{BS}$ is set as $P_{\max }=40 \mathrm{dBm}$, and the reflection coefficient of backscatter tag is set as $0 \leq \alpha_{\max } \leq 1$.

The results in Fig. 3 illustrate the sum-rate achieved by the proposed multi-cell NOMA BackCom scheme against the number of iterations when the number BSs is varied. The value of maximum BS power budget used is $P_{\max }=40 \mathrm{dBm}$. The result highlights that the proposed NOMA BackCom scheme has low complexity and converges within few iterations. For

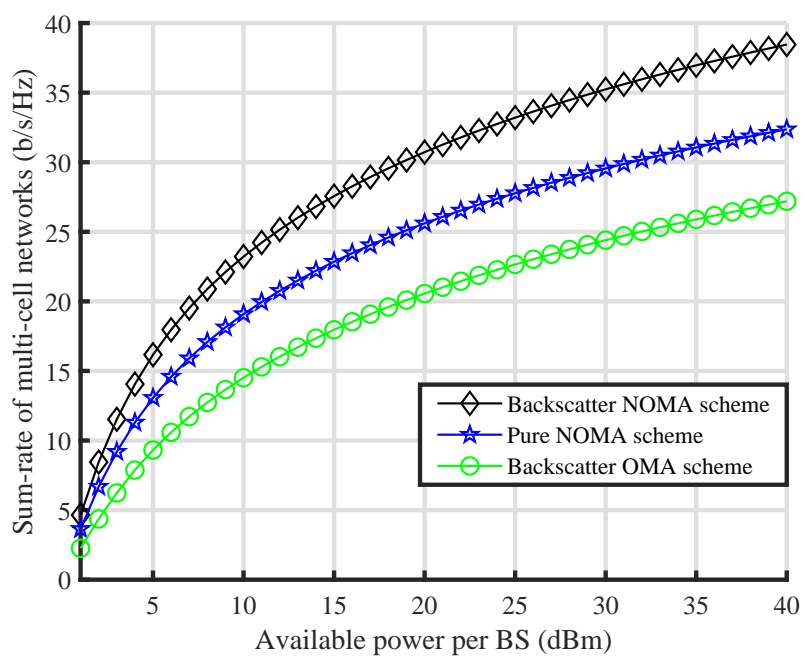

Fig. 4: Sum-rate of multi-cell BackCom versus available transmit power at each BS.

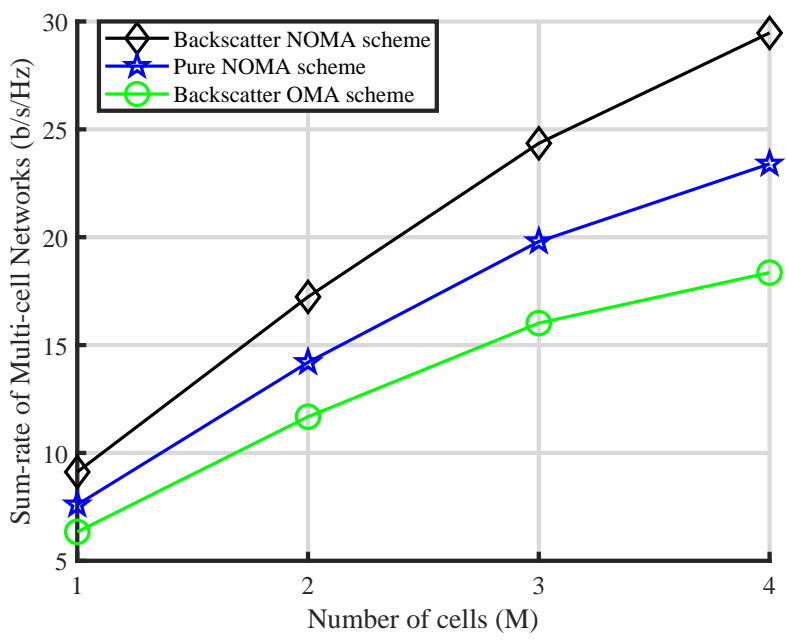

Fig. 5: Sum-rate of multi-cell BackCom versus number of cells in the network.

example, the sum-rate of the network for both cases remains unchanged after 10 iterations. Moreover, a system with more cells can achieve more sum-rate compared to the system with less number of cells. It is because the system with more cells can accommodate more NOMA user equipments and backscatter tags.

Now we present the sum-rate provided by the NOMA BackCom scheme in the multi-cell network as compared to the pure NOMA and OMA BackCom schemes. In this regard, Fig. 4 depicts the impact of BS available power budget on the system sum-rate when setting the number of BSs as $M=4$, and the backscatter tag's maximum reflection coefficient is considered as $\alpha_{\max }=1$. As expected, both NOMA and OMA schemes increase the system sum-rate when the total power budget of BS is increased. It can be seen from Fig. 4 that the NOMA BackCom technique outperforms the pure NOMA and conventioanl OMA schemes. For instance, when the transmit power of each BS is $P_{\max }=25 \mathrm{dBm}$, the sum-rate of 


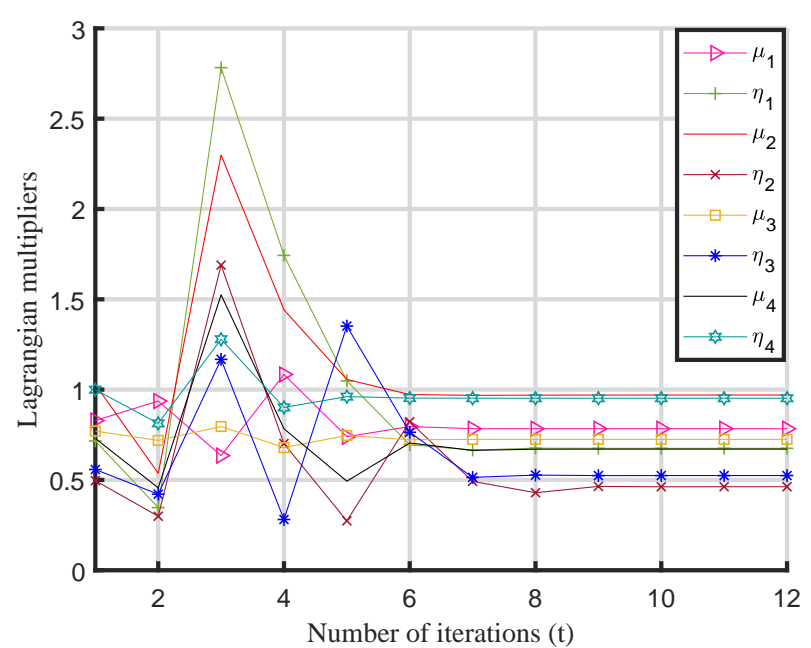

Fig. 6: Plot of number of iterations versus Lagrangian multipliers of the NOMA BackCom scheme.

our proposed NOMA BackCom scheme is $33.2 \mathrm{~b} / \mathrm{s} / \mathrm{Hz}$ in comparison to the benchmark pure NOMA and OMA schemes, i.e., 27.77 and $22.65 \mathrm{~b} / \mathrm{s} / \mathrm{Hz}$. Besides, the sum-rate of the proposed technique is significantly enhanced in comparison to the other two schemes in the case when transmission power of BS is increased. This is because the proposed NOMA based technique allocated more efficiently the extra available transmit power at BS. Moreover, the integration of BackCom with NOMA achieves significant gains.

In Fig. 5, the system sum-rate is plotted against the number of cells when the maximum power budget of each BS is $P_{\max }=40 \mathrm{dBm}$. As the available power budget for each BS is increased, the proposed NOMA BackCom scheme as well as other schemes achieve higher sum-rate. However, the performance of the NOMA BackCom scheme is significantly better than the pure NOMA and OMA schemes. More specifically, if the total BSs are 4, the sum-rate of NOMA BackCom is $36.41 \mathrm{~b} / \mathrm{s} / \mathrm{Hz}$. On the other hand, sum-rate achieved by the pure NOMA scheme is 30.37 , and OMA scheme provides a sumrate of 25.32. Furthermore, as the number of BSs varies from 1 to 4 , the sum-rate differennce between NOMA and OMA techniques is increased. It is because the capability of NOMA to accommodate more user equipments on the same network resources. These observations showed that using NOMA can significantly enhance the system performance and OMA is limited to achieve the high demands of the next-generation network.

Last but not the least, it is important to see the convergence behavior of the Lagrangian multipliers which is involved in the solution of the proposed optimization framework. It can be seen from Fig. 6 that the values of Lagrange multipliers against the iteration number. We can see that all the Lagrangian multipliers converge within limited number of iterations. This shows that the the proposed multi-cell NOMA BackCom technique exhibits low complexity.

\section{COnClusions}

NOMA BackCom is gradually maturing with every passing year. However, the performance gains of such systems are not well-explored in the existing literature. In this regard, this article has presented a resource allocation approach to enhance the spectral efficiency of the NOMA BackCom in a multi-cell environment. More specifically, a non-convex sumrate maximization problem has been formulated with various practical constraints. Subsequently, the problem is divided into two subproblems i.e., optimal reflection coefficient selection and BS power allocation. Both of these problems are then solved using a dual theory approach, and the results have been compared with the conventional OMA multi-cell networks. The simulation results clearly illustrate the performance gains obtained by using our proposed optimization framework.

Although the findings in this work can significantly contribute to the state-of-the-art, yet our work can be extended in various ways. For example, one critical issue in NOMA BackCom is that the reflected signal is broadcast and can be intercepted by a malicious eavesdropper in the network. To solve this issue, our work would be extended to improve the secrecy capacity and reduce the secrecy outage probability of such systems. Another challenge in NOMA BackCom in a multi-cell environment can be the appropriate selection of BS for reflecting the superimposed NOMA signal. Our optimization framework can also be extended to improving the selection of BS in a multi-cell environment. These open research topics will be investigated in the future.

\section{REFERENCES}

[1] C. De Alwis et al., "Survey on 6G frontiers: Trends, applications, requirements, technologies and future research," IEEE Open Journal of the Communications Society, vol. 2, pp. 836-886, 2021.

[2] W. U. Khan et al., "Joint spectral and energy efficiency optimization for downlink NOMA networks," IEEE Transactions on Cognitive Communications and Networking, vol. 6, no. 2, pp. 645-656, 2020.

[3] X. Li et al., "Residual transceiver hardware impairments on cooperative NOMA networks," IEEE Transactions on Wireless Communications, vol. 19, no. 1, pp. 680-695, 2020.

[4] K. Dev et al., "DDI: A novel architecture for joint active user detection and IoT device identification in grant-free NOMA systems for $6 \mathrm{G}$ and beyond networks," IEEE Internet of Things Journal, 2021.

[5] O. Maraqa et al., "A survey of rate-optimal power domain NOMA with enabling technologies of future wireless networks," IEEE Communications Surveys Tutorials, pp. 1-1, 2020.

[6] W. U. Khan et al., "Efficient power allocation in downlink multi-cell multi-user NOMA networks," IET Commun., vol. 13, no. 4, pp. 396402, 2018.

[7] W. U. Khan et al., "Spectral efficiency optimization for next generation NOMA-enabled IoT networks," IEEE Trans. Veh. Technol., vol. 69, no. 12, pp. 15 284-15297, Dec. 2020.

[8] X. Li et al., "Security analysis of multi-antenna NOMA networks under I/Q imbalance," Electronics, vol. 8, no. 11, p. 1327, 2019.

[9] Z. Ali et al., "Joint user pairing, channel assignment and power allocation in NOMA based CR systems," Applied Sciences, vol. 9, no. 20, p. 4282, 2019.

[10] L. Zhu et al., "Millimeter-wave communications with non-orthogonal multiple access for B5G/6G," IEEE Access, vol. 7, pp. 116 123-116132, 2019.

[11] A. Nasser et al., "Interference mitigation and power allocation scheme for downlink MIMO-NOMA HetNet," IEEE Transactions on Vehicular Technology, vol. 68, no. 7, pp. 6805-6816, July 2019.

[12] Y. Du et al., "Efficient multi-user detection for uplink grant-free NOMA: prior-information aided adaptive compressive sensing perspective," IEEE Journal on Selected Areas in Communications, vol. 35, no. 12, pp. 28122828, Dec 2017. 
[13] F. Jameel et al., "A comprehensive survey on cooperative relaying and jamming strategies for physical layer security," IEEE Communications Surveys Tutorials, vol. 21, no. 3, pp. 2734-2771, 2019.

[14] W. U. Khan et al., "Multiobjective optimization of uplink NOMAenabled vehicle-to-infrastructure communication," IEEE Access, vol. 8, pp. 84467-84 478, 2020.

[15] Z. Ding et al., "Impact of non-orthogonal multiple access on the offloading of mobile edge computing," IEEE Transactions on Communications, vol. 67, no. 1, pp. 375-390, 2018.

[16] C. Xu et al., "Practical backscatter communication systems for batteryfree internet of things: A tutorial and survey of recent research," IEEE Signal Process. Mag., vol. 35, no. 5, pp. 16-27, 2018.

[17] F. Jameel et al., "NOMA-enabled backscatter communications: towards battery-free IoT networks," IEEE Internet Things Mag., pp. 1-1, 2020.

[18] W. Liu et al., "Full-duplex backscatter interference networks based on time-hopping spread spectrum," IEEE Trans. Wireless Commun., vol. 16, no. 7, pp. 4361-4377, 2017.

[19] G. Wang et al., "Ambient backscatter communication systems: Detection and performance analysis," IEEE Trans. Commun., vol. 64, no. 11, pp. 4836-4846, 2016.

[20] W. U. Khan et al., "NOMA-enabled wireless powered backscatter communications for secure and green IoT networks," in Wireless-Powered Backscatter Communications for Internet of Things. Springer, 2020, pp. 103-131.

[21] X. Cao et al., "A distributed ambient backscatter MAC protocol for internet-of-things networks," IEEE Internet Things J., vol. 7, no. 2, pp. $1488-1501,2020$

[22] B. Lyu et al., "The optimal control policy for RF-powered backscatter communication networks," IEEE Trans. Veh. Technol., vol. 67, no. 3, pp. 2804-2808, 2017.

[23] J. Guo et al., "Backscatter communications with NOMA (Invited Paper)," in Int. Symp. Wireless Commun. Sys. (ISWCS), Aug. 2018, pp. $1-5$.

[24] F. Jameel et al., "Simultaneous harvest-and-transmit ambient backscatter communications under rayleigh fading," EURASIP J. Wireless Commun. Net., vol. 2019, no. 1, p. 166, 2019.

[25] F. Jameel et al., "Applications of backscatter communications for healthcare networks," IEEE Network, vol. 33, no. 6, pp. 50-57, 2019.

[26] X. Li et al., "Physical layer security of cognitive ambient backscatter communications for green Internet-of-things," IEEE Trans. Green Commun. Netw., pp. 1-1, 2021.

[27] Q. Tao et al., "A novel interference cancellation scheme for bistatic backscatter communication systems," IEEE Commun. Lett., pp. 1-1, 2021.

[28] Y. Xu et al., "Joint computation offloading and radio resource allocation in MEC-based wireless-powered backscatter communication networks," IEEE Trans. Vehic. Technol., pp. 1-1, 2021.

[29] Y. Wang et al., "Energy-efficient covert communications for bistatic backscatter systems," IEEE Trans. Vehic. Technol., vol. 70, no. 3, pp. 2906-2911, 2021.

[30] G. Sacarelo and Y. H. Kim, "Rate-energy tradeoffs of wireless powered backscatter communication with power splitting and time switching," IEEE Access, vol. 9, pp. 10 844-10 857, 2021.

[31] F. Jameel et al., "Time slot management in backscatter systems for largescale IoT networks," in Wireless-Powered Backscatter Communications for Internet of Things. Springer, pp. 51-65.

[32] A. Mehmood et al., "Preventing identity attacks in RFID backscatter communication systems: A physical-layer approach," in 2020 International Conference on UK-China Emerging Technologies (UCET). IEEE, 2020, pp. 1-5.

[33] F. Jameel et al., "Multi-tone carrier backscatter communications for massive IoT networks," in Wireless-Powered Backscatter Communications for Internet of Things. Springer, pp. 39-50.

[34] Q. Zhang et al., "Backscatter-NOMA: A symbiotic system of cellular and Internet-of-Things networks," IEEE Access, vol. 7, pp. 20000 $20013,2019$.

[35] J. Guo et al., "Design of non-orthogonal multiple access enhanced backscatter communication," IEEE Trans. Wireless Commun., vol. 17, no. 10, pp. 6837-6852, 2018.

[36] A. Farajzadeh et al., "UAV data collection over NOMA backscatter networks: UAV altitude and trajectory optimization," in IEEE Int. Conf. Commun. (ICC), May 2019, pp. 1-7.

[37] C. Le and D. Do, "Outage performance of backscatter NOMA relaying systems equipping with multiple antennas," IET Electron. Lett., vol. 55, no. 19 , pp. 1066-1067, 2019
[38] Y. Li et al., "Secure beamforming in MISO NOMA backscatter device aided symbiotic radio networks," arXiv preprint arXiv:1906.03410, 2019.

[39] G. Yang et al., "Resource allocation in NOMA-enhanced backscatter communication networks for wireless powered IoT," IEEE Wireless Commun. Lett., vol. 9, no. 1, pp. 117-120, 2020.

[40] S. Zeb et al., "NOMA enhanced backscatter communication for green IoT networks," in Int. Symp. Wireless Commun. Sys. (ISWCS), Aug. 2019, pp. 1-5.

[41] Q. Zhang et al., "Backscatter-NOMA: An integrated system of cellular and internet-of-things networks," in IEEE Int. Conf. Commun. (ICC), May 2019, pp. 1-6.

[42] W. U. Khan et al., "Secure backscatter communications in multi-cell NOMA networks: Enabling link security for massive IoT networks," in IEEE INFOCOM 2020-IEEE Conference on Computer Communications Workshops (INFOCOM WKSHPS). IEEE, 2020, pp. 213-218.

[43] — "Secure backscatter-enabled NOMA system design in 6G era," Internet Technology Letters, p. e307, 2021.

[44] F. Jameel et al., "Reinforcement learning for scalable and reliable power allocation in SDN-based backscatter heterogeneous network," in IEEE INFOCOM 2020 - IEEE Conference on Computer Communications Workshops (INFOCOM WKSHPS), 2020, pp. 1069-1074.

[45] W. U. Khan et al., "Joint Optimization of NOMA-enabled backscatter communications for Beyond 5G IoT networks," Internet Technol. Lett. p. e265.

[46] _- "Backscatter-enabled NOMA for future 6G systems: A new optimization framework under imperfect SIC," IEEE Commun. Lett., vol. 25 , no. 5, pp. 1669-1672, May 2021

[47] — "Backscatter-enabled efficient V2X communication with nonorthogonal multiple access," IEEE Trans. Vehic. Technol., vol. 70, no. 2, pp. 1724-1735, Feb. 2021

[48] Z. Zhang et al., "Downlink and uplink non-orthogonal multiple access in a dense wireless network," IEEE Journal on Selected Areas in Communications, vol. 35, no. 12, pp. 2771-2784, 2017.

[49] B. Xu et al., "Resource allocation in energy-cooperation enabled twotier NOMA HetNets toward green 5G," IEEE Journal on Selected Areas in Communications, vol. 35, no. 12, pp. 2758-2770, 2017.

[50] A. Ali et al., "Efficient resource management for sum capacity maximization in 5G NOMA systems," Applied System Innovation, vol. 2 no. 3, p. 27, 2019.

[51] F. Jameel et al., "Secrecy analysis and learning-based optimization of cooperative NOMA SWIPT systems," in 2019 IEEE International Conference on Communications Workshops (ICC Workshops). IEEE, 2019, pp. 1-6.

[52] J. Qian et al., "IoT Communications With $M$-PSK Modulated Ambient Backscatter: Algorithm, Analysis, and Implementation," IEEE Internet Things J., vol. 6, no. 1, pp. 844-855, 2018.

[53] D. Li and Y.-C. Liang, "Adaptive ambient backscatter communication systems with MRC," IEEE Trans. Veh. Technol., vol. 67, no. 12, pp. $12352-12357,2018$

[54] M. Chiang, "Nonconvex optimization for communication networks," in Advances in Applied Mathematics and Global Optimization. Springer, 2009, pp. 137-196.

[55] K. Bakht et al., "Power allocation and user assignment scheme for beyond 5G heterogeneous networks," Wireless Commun. Mobile Computing, vol. 2019, 2019.

[56] W. Aman et al., "Enhancing physical layer security in AF relayassisted multicarrier wireless transmission," Trans. Emerg. Telecommun. Technol., vol. 29, no. 6, p. e3289, 2018

[57] W. U. Khan et al., "NOMA-enabled optimization framework for nextgeneration small-cell IoV networks under imperfect SIC decoding," IEEE Transactions on Intelligent Transportation Systems, pp. 1-10, 2021 . 